\title{
Helium-filled soap bubbles for vortex core velocimetry
}

\author{
Giuseppe Carlo Alp Caridi ${ }^{1} \cdot$ Andrea Sciacchitano $^{1} \cdot$ Fulvio Scarano $^{1}$
}

Received: 18 November 2016 / Revised: 4 August 2017 / Accepted: 20 August 2017 / Published online: 30 August 2017

(C) The Author(s) 2017. This article is an open access publication

\begin{abstract}
Velocity measurements within the core of highswirl vortices are often hampered by heavier-than-air particle tracers being centrifuged outside the vortex core region. The use of neutrally buoyant and lighter-than-air tracers is investigated to aim at homogeneous tracers concentration in air flow experiments dealing with high-swirl vortices using particle image velocimetry. Helium-filled soap bubbles (HFSB) of sub-millimeter diameter are employed as flow tracers. Their density is controlled varying the relative amount of helium and soap solution composing the bubbles. The dynamics of HFSB and micro-size droplets is modeled within a Lamb-Oseen vortex to retrieve the order of magnitude of the tracers slip velocity. A positive radial drift for heavier-than-air tracers leads to an empty vortex core. In contrast, the concentration at the vortex axis is expected to increase for lighter than air tracers. Experiments are conducted on a sharp-edged slender delta wing at $20^{\circ}$ incidence. At chosen chord-based Reynolds numbers of $2 \times 10^{5}$ and $6 \times 10^{5}$, a stable laminar vortex is formed above the delta wing. Laser sheet visualization is used to inspect the spatial concentration of tracers. A comparison is made between micron-sized fog droplets and HFSB tracers in the nearly neutrally buoyant condition. Stereo-PIV measurements with fog droplets return a systematically underestimated axial velocity distribution within the vortex core due to drop-out of image cross-correlation signal. The nearly neutrally buoyant HFSB tracers appear to maintain a homogeneous spatial concentration and yield cross-correlation signal up to the
\end{abstract}

Giuseppe Carlo Alp Caridi

G.Caridi@tudelft.nl

1 Aerospace Engineering Department, TU Delft, Delft, The Netherlands vortex axis. The resulting velocity measurements are in good agreement with literature data.

\section{Introduction}

Vortex flow is a crucial research topic in aerodynamics. Several applications range from aircraft wing-tip vortices to those released by propellers or wind turbine blades. Wingtip and trailing-edge vortices are the most common examples of lift-induced vortices (Clancy 1975), responsible for the induced drag generated by the aircraft. Their prediction and control are crucial for instance in problems of air traffic management to account for the vortex-wake encounter problem around airports (Spalart 1998). In rotor aerodynamics, the vortex-blade interaction (VBI) is the main source of vibration and noise of helicopters (Conlisk 1997). The analysis of the tip vortex allows the determination of fundamental aerodynamic characteristics of wind turbines as the wake expansion, spiral vortex twist angle, and turbulent-kinetic mixing of the shear layer (Vermeer et al. 2003; Lignarolo et al. 2015). Furthermore, understanding the flow structure and the dynamic behavior of leading edge vortices (LEV) and fore body vortices is essential in the design of highly maneuverable slender bodied high speed aircrafts with delta wings (Nelson and Pelletier 2003).

Several studies have been reported in literature, which return the qualitative inspection of the vortex structure (Sohn and Chang 2012). Other works have quantitatively revealed complex flow features such as secondary vortices around delta wings, vortex breakdown and shear layer instabilities (Furman and Breitsamter 2013; Wang et al. 2016).

The flow field associated with vortical structures is fully three dimensional and often exhibits unsteady behavior. Under certain conditions, flow instabilities can induce the 
vortex breakdown with an escalation of its complexity, which makes it difficult to predict with numerical simulations (Ruith et al. 2003; Goto 2008). Experimental investigations are deemed essential not only for a direct characterization of the flow field, but also to support the development of vortex models and to validate numerical simulations (Devenport et al. 1996; Raffel et al. 2004; Bailey et al. 2006).

Particle image velocimetry (PIV) has been widely used to study vortex-dominated flows due to its ability to determine the velocity and vorticity distribution in a cross section of the vortex. Moreover, being non-intrusive (Raffel et al. 2007; Westerweel et al. 2013) PIV is well suited for the study of vortices emanating from rotating blades. Most PIV experiments conducted on vortices employed the planar approach based on the stereoscopic configuration (Konrath et al. 2013; Furman and Breitsamter 2013). One of the underlying hypotheses of particle image velocimetry is that the tracer particles can be assumed to move at the same velocity as the surrounding fluid. Detailed theoretical and experimental studies have led to quantitative criteria for the selection of particles for different fluid media and flow regimes (Melling 1997; Mei 1996).

Despite the fact that micrometric droplets have a response time in the order of microseconds and their Stokes number is well below $10^{-2}$ in low-speed flows, the systematic effect of a drifting force can lead to a significant lack of tracers in the core of a vortex. This unwanted phenomenon has been observed during several experiments conducted in the wake of rotors, delta wings and jet flows (Raffel et al. 2007). In these air flow experiments, di-ethyl-hexyl-sebacat (DEHS), oil or water-based droplets were used as tracers with typical diameter of $1 \mu \mathrm{m}$ and density in the order of $10^{3} \mathrm{~kg} / \mathrm{m}^{3}$. The high rotational velocity imparted to the particle tracers causes a slight, yet systematic outward drift due to the unbalance between the centrifugal force and the radial component of the pressure gradient. Such drift velocity is usually negligible for the purpose of velocity measurements; however, its overall effect depletes the inner region of the vortex. As a result, no velocity measurements can be obtained in the region with fewer to no particle tracers (1st international PIV challenge, Stanislas et al. 2003; Bhagwat and Ramasamy 2012). The use of even smaller particles can mitigate the problem of the radial drift; however, the amount of light scattered rapidly decays by decreasing the particle diameter (Adrian and Yao 1985; Lecuona et al. 2002).

Some investigations have been devoted to the errors introduced by the non-ideal behavior of the particle tracers in the vortex core. Leishman (1996) studied the effects of atomized olive oil droplets, in a tip vortex and concluded that the error on the radial velocity component is more significant than that on the tangential (i.e. swirling) component. Lecuona et al. (2002) underlined the problem caused by the lack of tracers in the vortex core and the dramatic drop of cross-correlation signal for PIV measurements. Clearly, the absence of signal is more deleterious than the error committed due to slip velocity between micron tracers and fluid.

The condition of neutral buoyancy (NB) as well as that of lighter-than-air (LTA) can be obtained in air flows using helium-filled soap bubbles (HFSB, Bosbach et al. 2009). The assessment of their suitability for wind tunnel experiments was recently given in the work of Scarano et al. (2015). The application to large scale aerodynamic problems using timeresolved tomographic PIV is documented in Caridi et al. (2016) who reported the measurement of the blade tip vortex region of a vertical axis wind turbine. In the latter work no evident deficit of particle concentration was observed in the core of the vortices emanating from the tip of the rotor blade.

The present work stems from the above observations and focuses upon the possibility to investigate vortex-dominated flows in wind tunnels avoiding the unwanted effect of tracers drop-out from the core region. To discuss the physical mechanism, a simplified and controllable condition with a steady laminar vortex was chosen. The specific condition of nearly neutral buoyancy and lighter-than-air for HFSB tracers is at the basis of the investigation. Tracers with a weight approaching that of the surrounding fluid are not expected to suffer from the mentioned radial outward drift because of the balance between centrifugal force and pressure gradient. Therefore, the tracers are expected to preserve a uniform concentration even within the highly swirling regions of the vortex.

The mechanical behavior of HFSB tracers is examined in a laminar vortex produced by a sharp-edged delta wing. The experiments take micron-sized droplets as a reference for the velocity measurements in the outer region of the vortex. Sub-millimeter HFSB tracers are produced with fine control of their relative weight from the lighter-than-air to the heavier-than-air condition. The visualization of the seeding concentration first gives an assessment of the importance of the radial drift in different conditions. Furthermore, the analysis of the time-averaged velocity field of the deltawing vortex as obtained with stereoscopic PIV quantifies the accuracy of the measurements with HFSB compared to the micron-sized droplets.

\section{Particle dynamics in a vortex}

The motion of a tracer particle immersed in a flow that features steady circular motion has been modeled in the work of Julien (1986) who studied the case of spherical particles moving in a Rankine vortex.

In the present section, first an order of magnitude analysis is conducted, that determines the tracers Stokes number. The latter is followed by a more detailed evaluation of the 
drifting motion of the tracer immersed in a vortex based on the governing equations describing the motion of a small tracer.

A direct, yet approximate, estimate of the adequacy of particle tracers in describing the fluid motion in a vortex can be based on the particle Stokes number $S t_{\mathrm{k}}$, the governing non-dimensional parameter for fluid-particles interaction. The latter is defined as the ratio between the characteristic response time of the particle to step-like velocity variation $\tau_{\mathrm{P}}$ and the time scale representative of the flow under analysis $\tau_{\mathrm{F}}$. Following the work of Samimy and Lele (1991) values of $S t_{\mathrm{k}}$ below $10^{-1}$ indicate that the tracers will follow the velocity fluctuations to within $1 \%$ discrepancy.

According to Rockwell (1993), the main time scale in a leading edge vortex released by a delta wing can be defined as the turn-over time: one local revolution of the vortex. Therefore, the characteristic time scale $\tau_{\mathrm{F}}$ can be associated with the turn-over time $\tau_{\text {to }}$, defined by the local value of the tangential velocity $U_{\mathrm{t}}$ and the radial position $r$, expressed as follows:

$\tau_{\mathrm{to}}=\frac{2 \pi r}{U_{\mathrm{t}}}$.

The present experiments are conducted at free stream velocity of 10 and $30 \mathrm{~m} / \mathrm{s}$. Knowing that the maximum tangential velocity of the LEV is approximately equal to $U_{\text {inf }}$ (Nelson and Pelletier 2003), a Lamb-Oseen vortex with a $U_{\mathrm{t} \text { max }}=U_{\text {inf }}$ is considered for the evaluation of the Stokes number along the radial position (Fig. 1). In a previous work from the authors, the particles characteristic time response has been experimentally determined (Scarano et al. 2015), and it ranges from 10 to $50 \mu$ s. It is worth remarking that the conservative assumption of a particle response time of $50 \mu \mathrm{s}$ is taken.

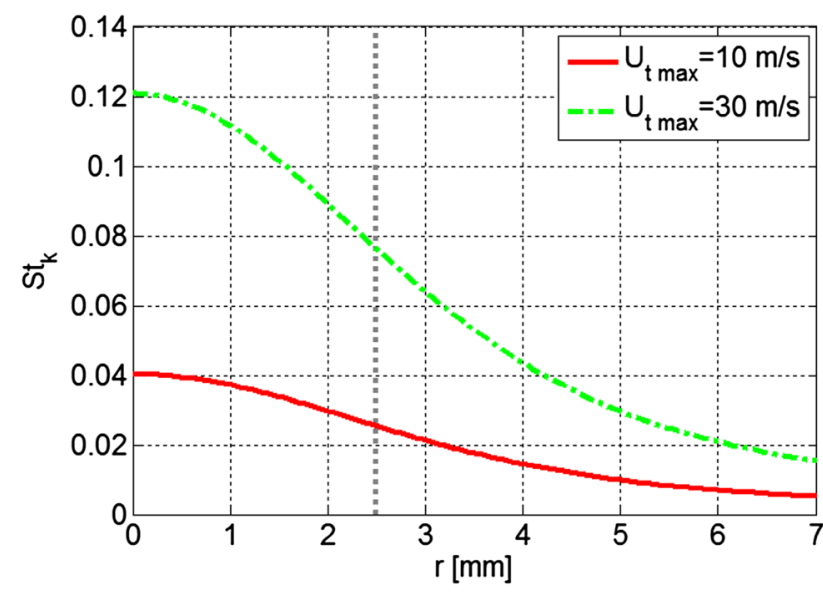

Fig. 1 Stokes number in a Lamb-Oseen vortex for HFSB tracers, vortex radius indicated with dashed gray line
The particles Stokes number lies between $10^{-2}$ and $10^{-1}$ indicating that HFSB tracers particle are potentially suited to measure the velocity in this type of flow. Although the Stokes number pertaining micrometric droplets is significantly smaller (their response time is at least one order of magnitude below that of HFSB), their systematic drift causes the core depletion.

Recalling the work of Julien (1986), in the frame of reference that travels with the fluid parcel surrounding the tracer, three forces act onto the particle: the centrifugal force that results from the particle (tangential) velocity $V$ along the curved trajectory, the pressure force $F_{\mathrm{p}}$, and the steady state drag force $F_{\mathrm{D}}$ due to the particle drifting radially with velocity $V_{\mathrm{r}}$ (Fig. 2 ).

The equation of motion is briefly recalled here:

$m_{\mathrm{p}} \frac{V^{2}}{r}=F_{\mathrm{D}}+F_{\mathrm{P}}$

where $m_{\mathrm{p}}$ is the tracer mass and $r$ is the radius of the circular trajectory. The velocity of the fluid $U$ is acting only on the tangential direction and it is assumed that $V_{\mathrm{t}}=U$. The only relevant component of the velocity difference between the particle and the surrounding fluid velocity is the radial one, where tracers motion lead to depletion of the concentration in the core. Therefore, the radial particle velocity $V_{\mathrm{r}}$ corresponds to the velocity slip $V_{\text {slip }}$. The latter can be obtained expressing the terms in Eq. (2) as:

$\frac{\pi}{6} d_{\mathrm{p}}^{3} \rho_{\mathrm{p}} \frac{U^{2}}{r}=3 \pi v d_{\mathrm{p}} \rho_{\mathrm{f}} V_{\text {slip }}+\frac{\pi}{6} d_{\mathrm{p}}^{3} \rho_{\mathrm{f}} \frac{U^{2}}{r}$.

Here, $\rho_{\mathrm{p}}$ and $\rho_{\mathrm{f}}$ are the density of the particle and of the fluid, respectively, and $v$ is the kinematic viscosity of the fluid. The transient effect of particle radial acceleration

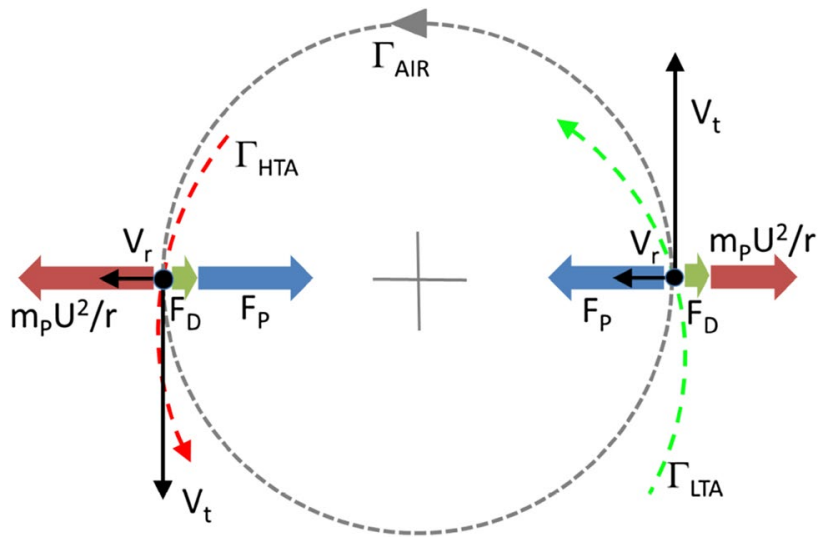

Fig. 2 Trajectory of heavier-than-air $\left(\Gamma_{\mathrm{HTA}}\right.$, dashed red $)$ and lighterthan-air $\left(\Gamma_{\mathrm{LTA}}\right.$, dashed green $)$ tracer within a flow with circular motion $\left(\Gamma_{\mathrm{AIR}}\right)$. Radial forces are indicated by thick arrows following definitions in Eq. (2). Tracer velocity decomposed into radial $\left(V_{\mathrm{r}}\right)$ and tangential $\left(V_{\mathrm{t}}\right)$ components 
is not considered here. Therefore, the slip velocity corresponds to the limit radial velocity attained by the particle subject to a constant radial acceleration. The expression of $V_{\text {slip }}$ is obtained from Eq. (3):

$V_{\text {slip }}=\frac{1}{18} \bar{\rho} \frac{U^{2}}{r v} d_{\mathrm{p}}^{2}$

where the normalized density difference $\bar{\rho}$ is given by:

$\bar{\rho}=\frac{\rho_{\mathrm{p}}-\rho_{\mathrm{f}}}{\rho_{\mathrm{f}}}$.

Equation (4) states that for a neutrally buoyant tracer (with $\bar{\rho}=0$ ), the slip velocity vanishes, with the particle exhibiting a circular trajectory. A micrometric droplet (with $\bar{\rho} \sim 10^{3}$ ) will slightly move outward from the vortex core region. Lighter-than-air tracers $(\bar{\rho}<0)$ move in the opposite direction converging towards the center of rotation: the vortex axis. The magnitude of the radial velocity increases linearly with the deviation from the neutrally buoyant condition, while it is proportional to the second power of the particle diameter and is highest at the edge of the vortex core where the flow exhibits high angular velocity. The latter consideration is relevant for the comparison between HFSB and micron-size droplets, because the former approximately satisfies the neutral buoyancy condition $(\bar{\rho} \cong 0)$; however, their diameter is two orders of magnitude larger.
A numerical evaluation of Eq. (4) is given here to yield an order-of-magnitude analysis for the radial slip of tracer particles within the Lamb-Oseen vortex model with core radius $r_{\mathrm{c}}=2.5 \mathrm{~mm}$ and maximum tangential velocity $U_{\max }=10 \mathrm{~m} / \mathrm{s}\left(\right.$ at $r=r_{\mathrm{c}}$ ). These conditions mimic those encountered in the present experiments with a free stream velocity $U_{\text {inf }}=10 \mathrm{~m} / \mathrm{s}$ (see Sect. 4). Fog droplets with a density of $1000 \mathrm{~kg} / \mathrm{m}^{3}(\bar{\rho} \cong 830)$ and $1 \mu \mathrm{m}$ in diameter are taken for reference of typical tracers employed in PIV. Helium-filled soap bubbles of $300 \mu \mathrm{m}$ diameter and five different buoyant conditions are considered following the previous work of Scarano et al. (2015). Figure 3 shows the radial distribution of the slip velocity for the chosen tracers. Despite the HFSB are two orders of magnitude larger than fog droplets, their low density allows them to recede from the vortex center with a velocity comparable to the fog droplets. The results indicate that HFSB with a control of their neutral buoyancy within $1 \%(\bar{\rho} \approx 0.01)$ yield the same radial slip as the fog droplets. The cases of Heliumfilled-soap-bubble within 0.5 and $5 \%$ departure from neutral buoyancy $\left(\bar{\rho}=5 \times 10^{-3}\right.$ and $\left.\bar{\rho}=5 \times 10^{-2}\right)$ are illustrated in the example. Close to the neutral buoyancy condition HFSB have not only similar behavior than the micron-size droplets, but most importantly, the lighter-than-air tracers exhibit a negative (centripetal) drift velocity. As a result, these tracers are expected to accumulate towards the vortex axis where the concentration is maintained high.

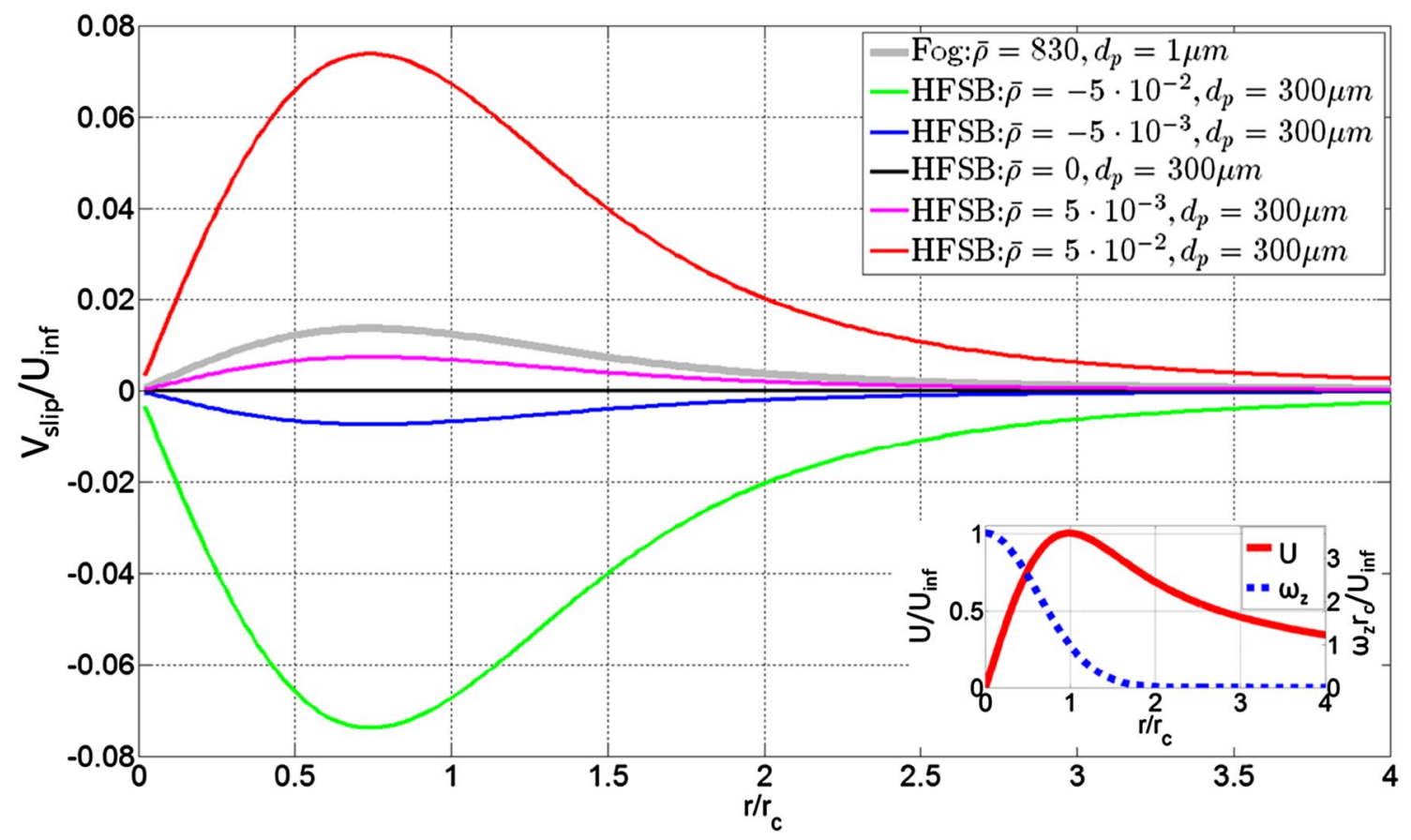

Fig. 3 Tracer slip velocity for different seeding particles, with radial profiles of dimensionless velocity of the flow, $U$, and vorticity, $\omega_{z}$, for a Lamb-Oseen vortex 
The analysis of the effect of finite size of HFSB tracers in conjunction with the pressure distribution in the vortex core yields negligible deviations of the particle motion with respect to the case of a point-wise tracer. For sake of conciseness, further discussion is included in Appendix A for the interested reader.

\section{Experimental apparatus and measurement procedure}

The experiments are conducted in a low-speed open test section wind tunnel (W-tunnel) of the Aerospace Engineering aerodynamic laboratories of TU Delft. The W-tunnel features a test section of $40 \times 40 \mathrm{~cm}^{2}$ and the air flow reaches $30 \mathrm{~m} / \mathrm{s}$ with a turbulence level below $1 \%$.

A sharp-edge $76^{\circ}$ swept delta wing is used with chord length of $300 \mathrm{~mm}$. The model is made of acrylic glass (PMMA) and painted black to minimize the laser light reflections. The bottom side of the leading edge is beveled at $30^{\circ}$. Figure 4 shows the details of the model (left) and its installation at the exit of the wind tunnel (right) at $20^{\circ}$ incidence and a free stream flow velocity $U_{\text {inf }}$ varying from 10 to $30 \mathrm{~m} / \mathrm{s}$. The blockage ratio generated by the model is $4.6 \%$. The corresponding chord-based maximum Reynolds number is $R e_{\mathrm{c}}=6 \times 10^{5}$.

Laser sheet visualization is performed in a plane perpendicular to the surface of the delta wing that captures the entire vortex region at approximately $75 \%$ of the chord. A Photron FastCAM SA1 camera (CMOS, $1024 \times 1024$ pixels, $20 \mu \mathrm{m}$ pixel pitch, $5400 \mathrm{fps}$ ) records the light scattered by the tracers from a distance of $2.4 \mathrm{~m}$. The active area of the sensor is cropped to $512 \times 512$ pixels. At the set magnification of $M=0.13$, the field of view is $15 \mathrm{~cm}$ and covers the wing span.
The imaging apparatus used for the stereoscopic PIV makes use of two cameras of the same type as above. The cameras are equipped with $200 \mathrm{~mm}$ focal length Nikon objectives $\left(f_{\#}=16\right)$ mounted on Scheimpflug adapters. The angle between the cameras is set to $45^{\circ}$. A magnification factor $(M=0.25)$ yields a digital imaging resolution of 18.7 pixels $/ \mathrm{mm}$. The field of view captures the leading edge vortex on the right side of the wing. The system of coordinates chosen for the present study is illustrated in Fig. 4, right, with the $z$-axis along the model chord, the $x$ - and $y$-axes along the span and wall-normal directions, respectively.

To determine the time-averaged scattering intensity of tracers, 40,000 images are recorded at 250 frames per second for each type of seeding. For the stereoscopic-PIV measurements, sequences of 40,000 double-frame images are acquired with time separation of $\Delta t=60 \mu$ s for a free stream of $10 \mathrm{~m} / \mathrm{s}$ and $\Delta t=10 \mu \mathrm{s}$ for a free stream of $30 \mathrm{~m} / \mathrm{s}$. The analysis of the stereoscopic recordings is carried out within LaVision Davis 8.3. Given the relatively high imaging signal-to-noise ratio yielded by the HFSB tracers and the absence of solid surface in the field of view, no image pre-processing was required for background intensity. The cross-correlation analysis is based on iterative multigrid window deformation. A starting window size of $96 \times 96$ pixels is selected and progressively refined to $32 \times 32$ pixels with overlap factor of $50 \%$. The final vector pitch is $0.85 \mathrm{~mm}$. The seeding density for the HFSB experiments is extremely low (typically no more than 10 particles are present in each recording). Therefore, the overall ensemble of 40,000 images has been analyzed with the ensemble correlation algorithm (Meinhart et al. 2000).
Fig. 4 Left top and side views of the delta wing model. Right experimental arrangement with the model installed at the wind tunnel exit. The field of view (FOV) of PIV measurements is indicated by red dashed lines. Time-averaged image of planar illumination using HFSB with lighter-than-air condition
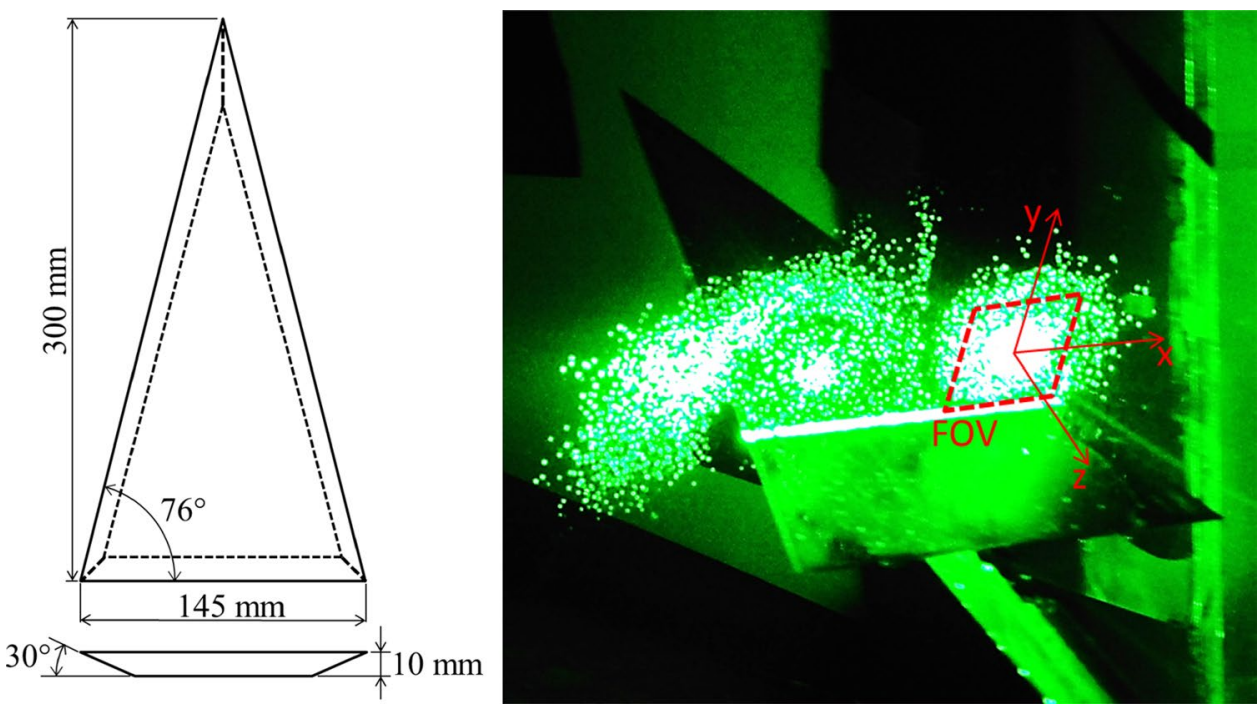


\subsection{Flow seeding}

The micron-sized droplets are based on a water-glycol solution vaporized with a SAFEX fog generator. The mean droplets diameter is $1 \mu \mathrm{m}$ with a density of approximately $1000 \mathrm{~kg} / \mathrm{m}^{3}$. HFSB are generated by an orifice-type bubble generator similar to that described by Bosbach et al. (2009). The fluids supply unit (FSU) that controls the flow rate of soap fluid, helium, and air is schematically represented in Fig. 5 and is provided by LaVision GmbH. The unit has the function of controlling the flow rate of helium, soap fluid, and air by setting the pressure upstream of the supply line. The air is provided by a pressurized facility and the helium from a pressurized vessel.

Generation of neutrally buoyant (NB), lighter-than-air (LTA), and heavier-than-air (HTA) bubbles relies on a dedicated adjustment of the flow rates of $\mathrm{He}$ and bubble fluid solution (BFS). The relaxation time of NB bubbles, used for PIV measurements, ranges between 10 and $30 \mu$ s (Scarano et al. 2015). The size of the bubbles is mainly controlled by the air flow rate, and it is determined from the raw images measuring the distance between glare points. The latter procedure yields an estimate of the mean diameter of $300 \mu \mathrm{m}$. The uncertainty of the diameter estimation is approximately $30 \mu \mathrm{m}$ (see Fig. 6).

The fog generator is positioned upstream the fan of the wind tunnel to allow a homogeneous seeding distribution. Instead, the bubble generator is placed inside the settling chamber approximately $1.2 \mathrm{~m}$ upstream of the contraction and $2 \mathrm{~m}$ from the delta wing. An assessment of the free stream turbulence in the test section with the presence of the bubble generator yields practically the same level of rms fluctuations as with the empty settling chamber.

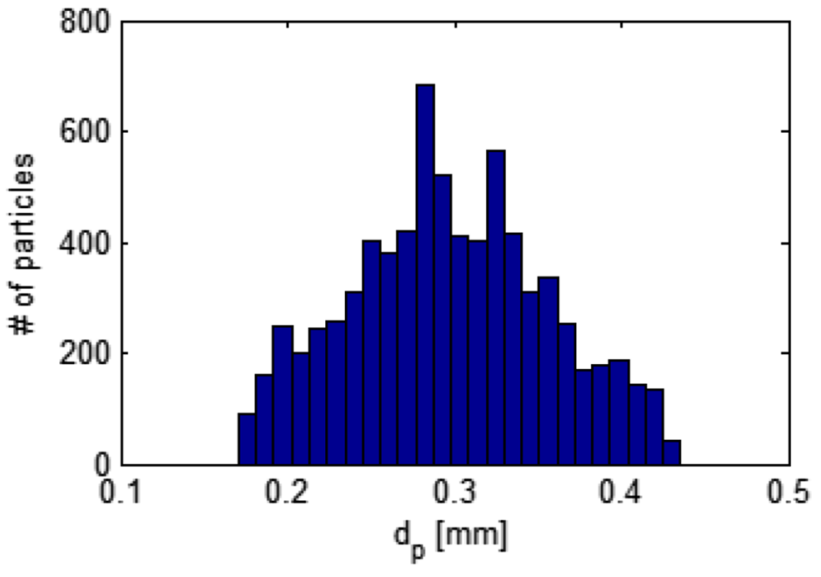

Fig. 6 NB-HFSB size distribution of the PIV measurements

\section{Tracers visualization within leading edge vortex}

Experiments conducted with laser sheet visualization aim at determining the tracers concentration in the vortex region above the delta wing. The time-average scattered light intensity from micron-sized droplets yields a rather uniform concentration with a clear deficit region centered around the axis of the vortex. The extent of the circular deficit region is approximately $5 \mathrm{~mm}$ in diameter (Fig. 7a). The latter corresponds approximately to the vortex core radius as discussed in the remainder. Increasing the free stream velocity from 10 to $30 \mathrm{~m} / \mathrm{s}$ does not alter the situation significantly (Fig. 7d). However, from instantaneous snapshots, the deficit region slightly grows with the flow velocity (Fig. 7b, e), which is expected from the increase of the radial drift with the square of the tangential velocity.

The experiments conducted with HFSB in the nearly neutrally buoyant condition $(|\bar{\rho}|<0.01)$ also yield an overall uniform concentration of the tracers (Fig. 7c). Despite
Fig. 5 Sketch of supply unit for HFSB generators provided by LaVision $\mathrm{GmbH}$

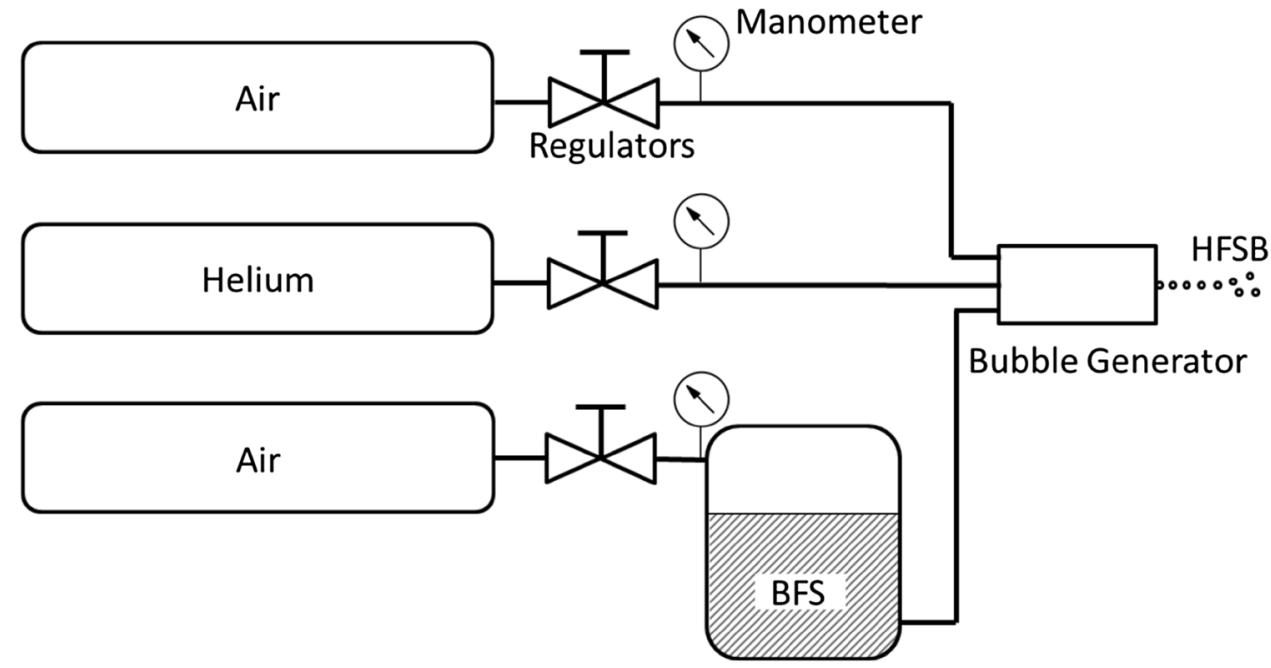




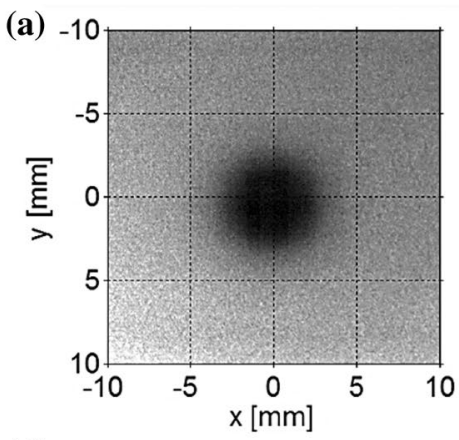

(d)

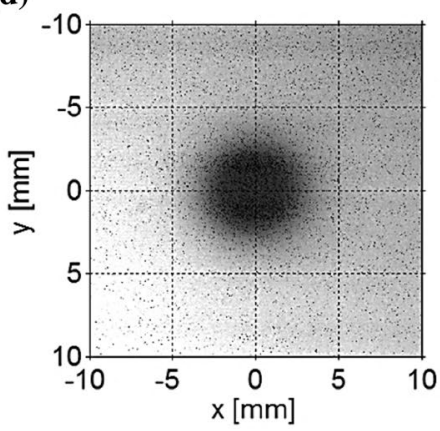

(b)

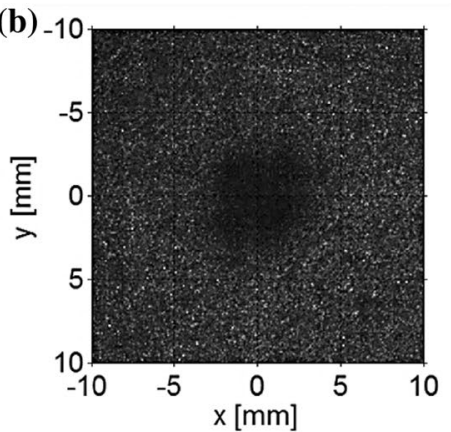

(e)

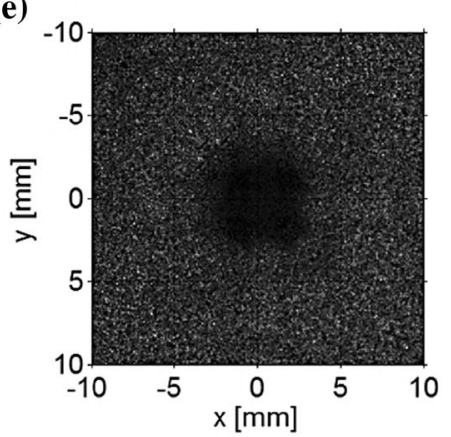

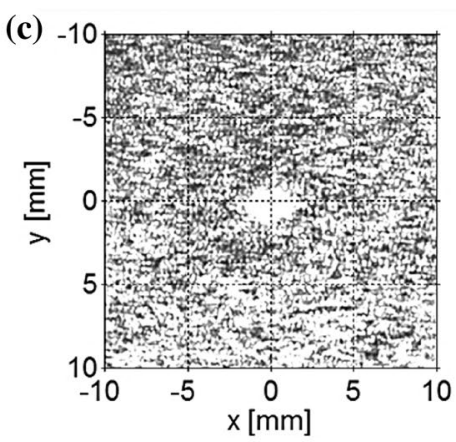

(f)

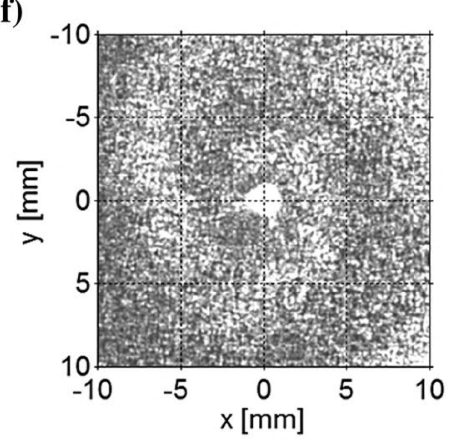

Fig. 7 Laser sheet visualization in the LEV: time-average recorded intensity with fog at $10 \mathrm{~m} / \mathrm{s}(\mathbf{a})$ and $30 \mathrm{~m} / \mathrm{s}$ (d); instantaneous particle images with fog at $10 \mathrm{~m} / \mathrm{s}(\mathbf{b})$ and $30 \mathrm{~m} / \mathrm{s}(\mathbf{e})$; time-average recorded intensity with HFSB at $10 \mathrm{~m} / \mathrm{s}(\mathbf{c})$ and $30 \mathrm{~m} / \mathrm{s}$ (f)

the large number of recordings, a fully uniform intensity is not achieved due to the small number of tracers (approximately 10) recorded in each image. The latter effect is more pronounced at higher flow velocity as the tracers concentration scales inversely proportional to it (Fig. 7f), as it is documented in a previous work of the authors (Caridi et al. 2016). This result is consistent with Eq. (3), which states that tracers with density close to that of the fluid experience less radial drift than the fog droplets. Furthermore, a small increase of seeding concentration is found at the core axis (Fig. 7c, f). This is ascribed to the fact that even a fraction of the bubbles population with lighter-than-air weight will experience a negative radial force and associated motion, resulting in accumulation of tracers around the vortex axis.

From a side view, the visualization of the tracers illustrates the presence of lighter-than-air tracers at the vortex axis resulting from their slight inward drift. Figure 8 shows a visualization of the tracers pathlines around the delta wing. The photograph is taken with exposure time of $5 \mathrm{~ms}$, while the laser is firing at $1 \mathrm{kHz}$ rate. The axis of the vortex where more particles accumulate is highlighted with a dashed red line. The continuous shape of the vortex axis indicates a quasi conical development of the vortex up till the trailing edge of the delta wing. Downstream, the vortex line bends
Fig. 8 Visualization of HFSB tracers pathlines by multipleexposure image $\left(U_{\text {inf }}=10 \mathrm{~m} / \mathrm{s}\right)$

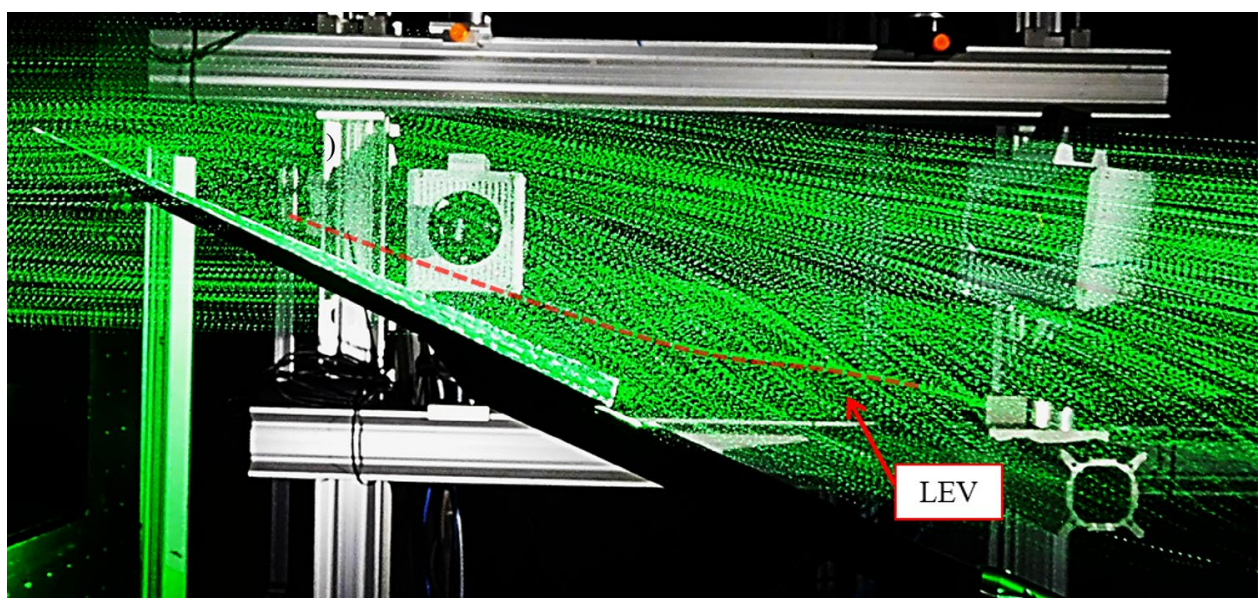


upwards; towards the edge of the photograph, the inception of vortex breakdown is signaled by a sudden broadening of the tracers at the axis.

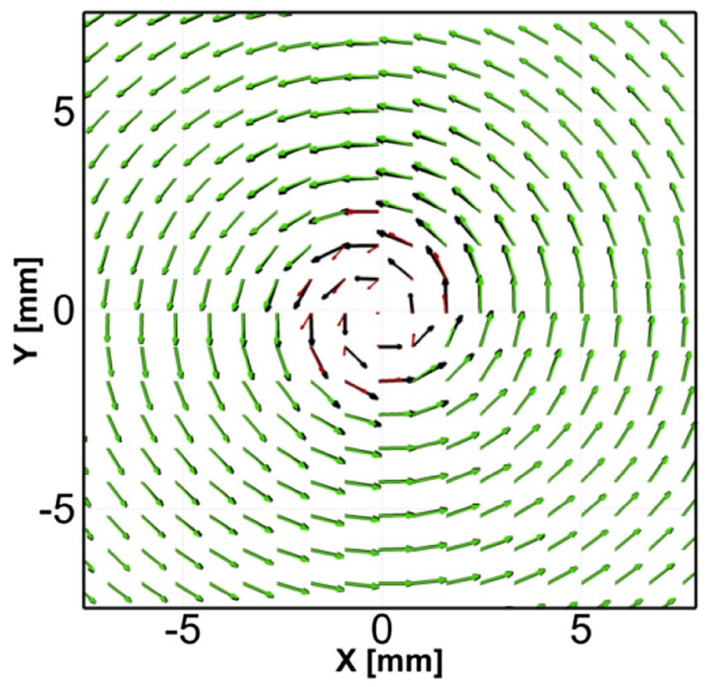

Fig. 9 Velocity vector field from measurements with HFSB and micro-size droplets at $10 \mathrm{~m} / \mathrm{s}$. Black vectors HFSB; green vectors micro-size droplets with $\mathrm{SNR}>1.5$; red vectors micro-size droplets with SNR $<1.5$

\section{Velocity field in the vortex region}

The time-average velocity fields obtained with fog droplets and HFSB are compared in Fig. 9. The measurement conducted with micro-size droplets suffers from loss of correlation signal in the core region, with a signal-to-noise ratio (SNR) well below a criterion for reliable velocity vector detection. In the core region the condition of SNR $<1.5$ is experienced and the estimates of the three velocity components are expected to be erroneous there. Outside of the core region, the measurements obtained with fog droplets follow with good agreement that of HFSB, which confirms the tracing fidelity of the latter.

The contours of the time-averaged tangential velocity field, measured with micro-size droplets and HFSB, are separately shown in Fig. 10 at the two values of the free stream velocity. The distribution of the tangential velocity features the expected ring shape and an annular region of maximum is observed at a radial distance of approximately $2.5 \mathrm{~mm}$ from the vortex axis. It is noted that only the inner part of the core differs between the measurements obtained with fog droplets and HFSB. In particular, the tangential velocity measured with fog droplets (Fig. 10a) exhibits several spurious measurement points (SNR $<1.5$, masked in white) and the velocity does follow a linear distribution as
Fig. 10 Color contours of time-averaged tangential velocity component. Fog droplets at $U_{\text {inf }}=10 \mathrm{~m} / \mathrm{s} \mathrm{(a)} \mathrm{and} 30 \mathrm{~m} / \mathrm{s}$ (c); HFSB at $U_{\text {inf }}=10 \mathrm{~m} / \mathrm{s}(\mathbf{b})$ and $30 \mathrm{~m} / \mathrm{s}(\mathbf{d})$ (a)

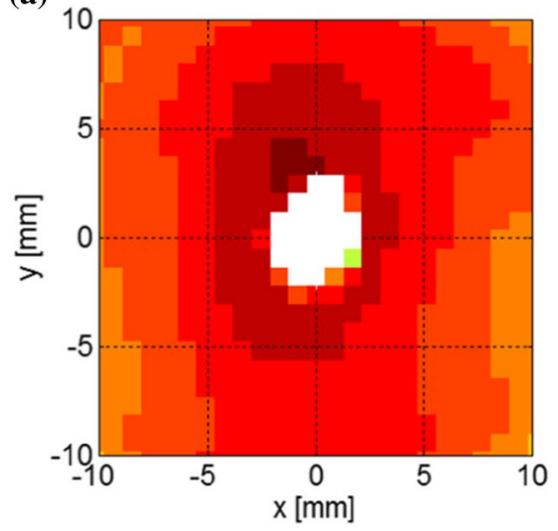

(c)

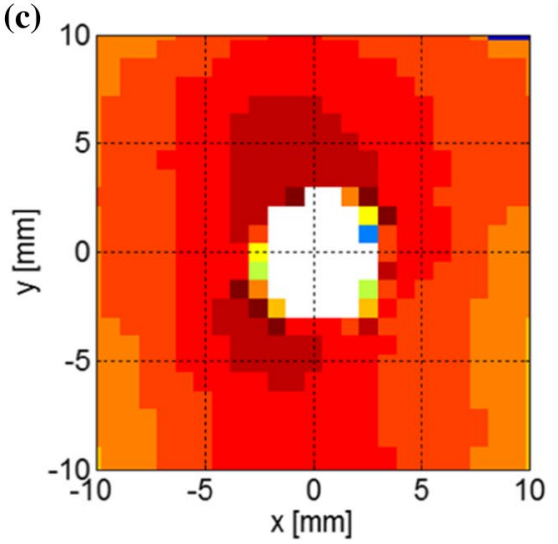

(b)

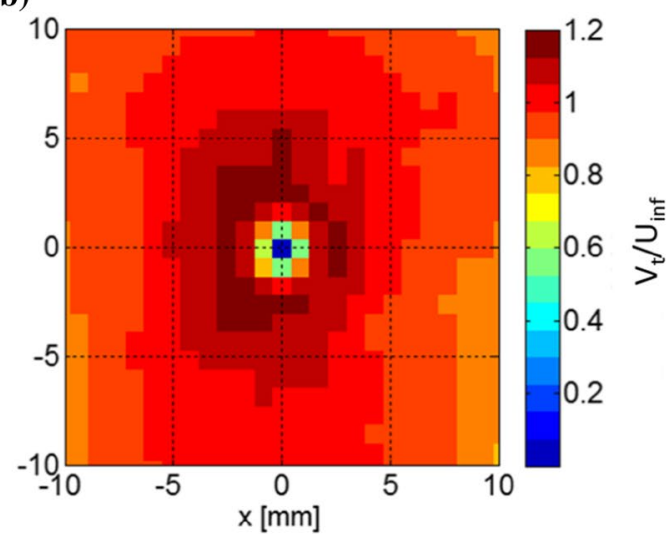

(d)

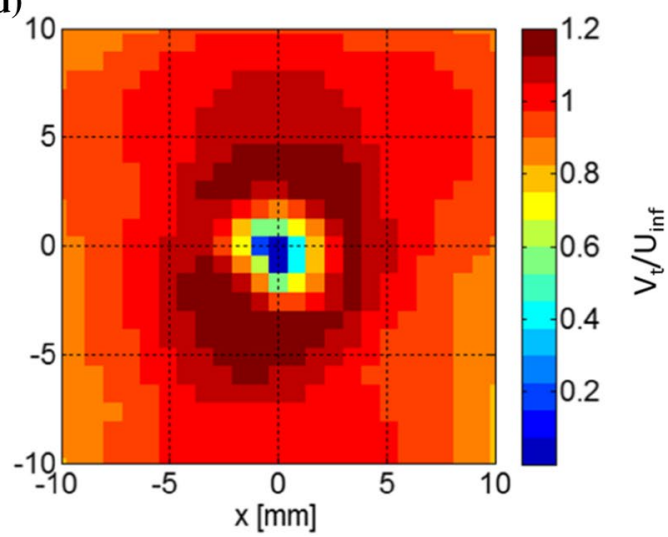


expected and documented in literature (Nelson and Pelletier 2003). The above effects are more pronounced at higher velocity (Fig. 10c) due to the increased effect of particle centrifugation. The measurements from HFSB (Fig. 10b, d) return a higher value of the maximum tangential velocity $\left(V_{\mathrm{t}}=1.2 U_{\text {inf }}\right)$ as well as a well-defined vortex center at velocity close to zero.

The axial velocity in the vortex region typically features a maximum or a minimum at the axis. This is reported as jetlike or wake-like behavior of the vortex (Nelson and Pelletier 2003). Excess or defect of axial velocity in the vortex core is an important property when predicting the occurrence of vortex breakdown (Escudier 1988). Therefore, the accurate measurement of the axial velocity and its streamwise development is relevant to the study of vortex-dominated flows. In the present case, a jet-like distribution is expected in the core of the vortex, which is revealed with the measurements done with HFSB (Fig. 11b). A self-similar behavior is observed when the axial velocity is normalized with the free stream value (Fig. 11b, d). The non-linear distribution of the axial velocity makes it impossible to estimate it from spatial interpolation of the values in the neighborhood of the vortex axis, which is the typical case when dealing with data drop-out in the center region of the vortex (Fig. 11a, c). The axial velocity peak value obtained with the HFSB measurements is approximately twice the free stream velocity, which agrees well with the previous experimental results reported by Nelson and Pelletier (2003).

A quantitative inspection of the velocity distribution in the vortex core region is presented by velocity profiles extracted across the vortex axis $(y=0 \mathrm{~mm})$. The distribution of the tangential velocity features the characteristic antisymmetric shape with a quasi-linear region around the center and two opposite extrema that identify the edge of the vortex core (Fig. 12, top). The core radius, defined as half of the peak-to-peak distance, is estimated to be $r_{\mathrm{c}}=2.5 \mathrm{~mm}$. The peak value measured with fog slightly underestimates that returned with HFSB (by approximately 10\%). Since the tangential velocity follows a linear distribution within the vortex core, the lack of signal caused by the fog tracers has limited consequences on the accuracy of this component. In fact, the tangential velocity in the vortex core could be evaluated by linear interpolation of velocity values at the edge of the core. The uncertainty stems from the underestimation of the peak value and its offset from the actual position.

Conversely, the absence of seeding has a non-negligible effect in the axial velocity component, where the lack of signal precludes the accurate estimation of the velocity peak, as it is shown in Fig. 12, bottom. Neither a spatial interpolation can be used to alleviate the strong bias (underestimation)
Fig. 11 Contour of the timeaveraged axial component of the velocity for a fog at $10 \mathrm{~m} / \mathrm{s}$; b HFSB at $10 \mathrm{~m} / \mathrm{s} ; \mathbf{c}$ fog at $30 \mathrm{~m} / \mathrm{s}$; d HFSB at $30 \mathrm{~m} / \mathrm{s}$ (a)

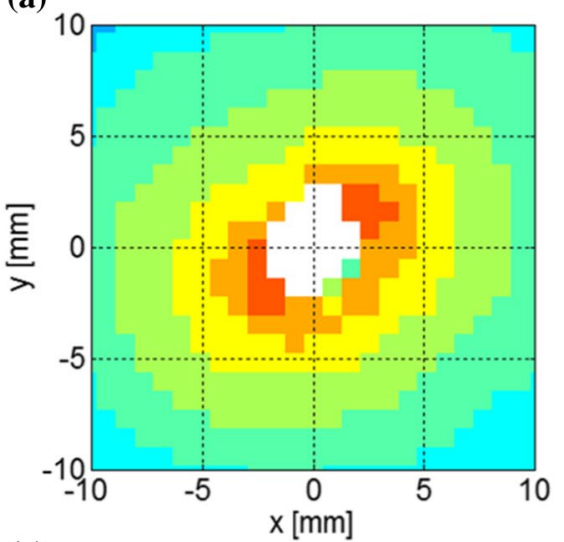

(c)

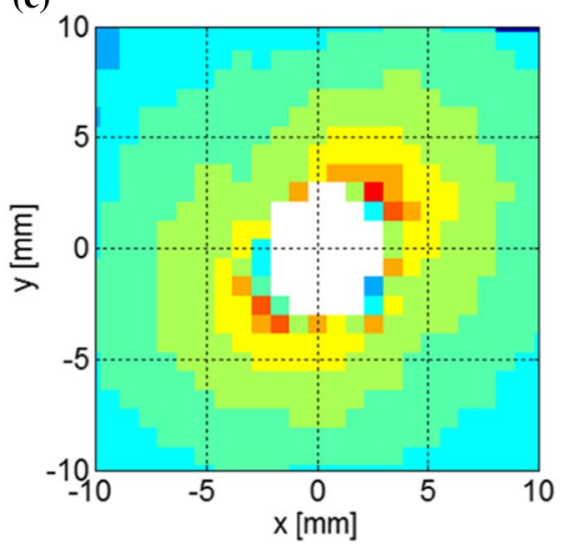

(b)

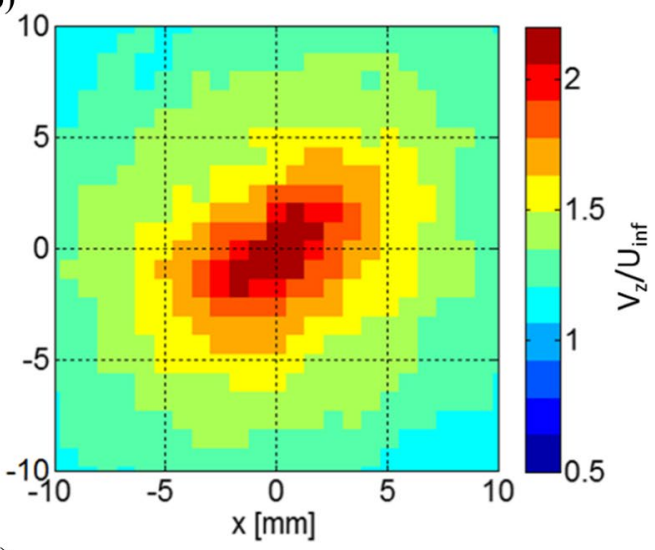

(d)

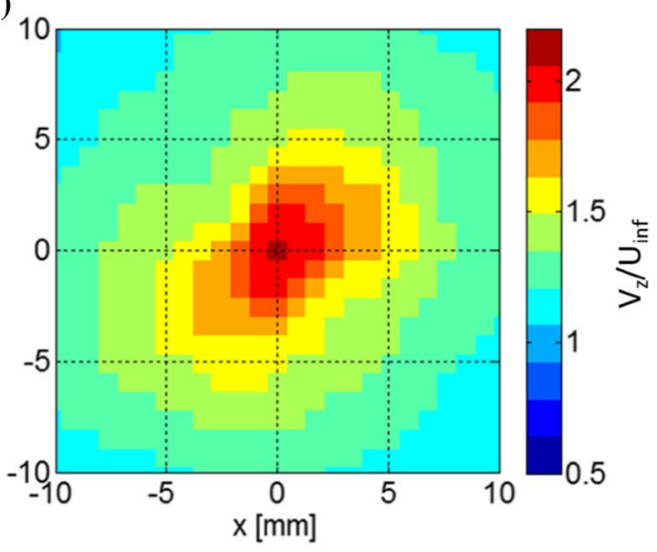



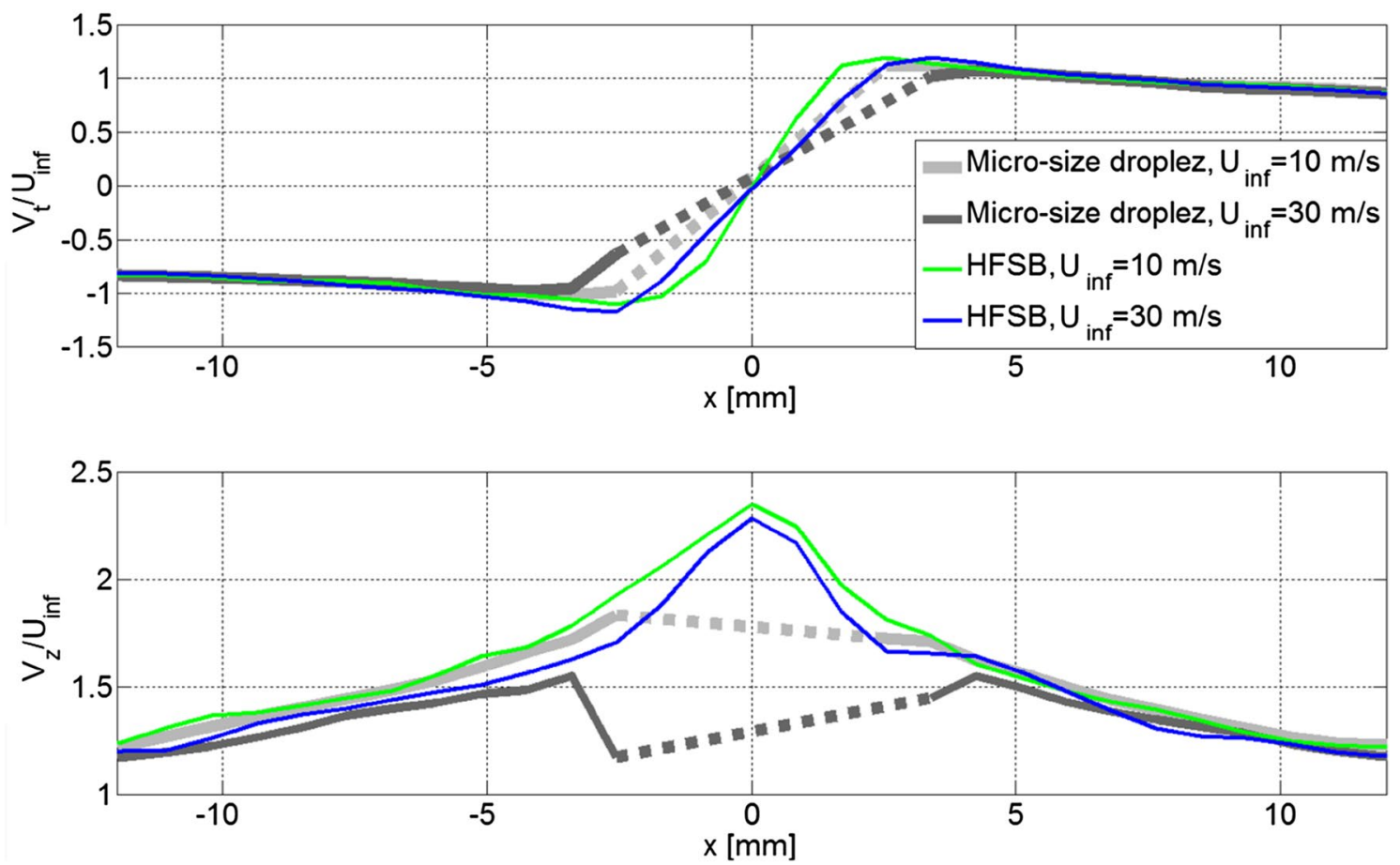

Fig. 12 Time-averaged velocities for different seeding particles at $U_{\text {inf }}=10 \mathrm{~m} / \mathrm{s}$. Top tangential velocity. Bottom axial velocity

that affects the axial velocity. Outside of the vortex core, the good correspondence between HFSB velocity and that of fog droplets confirms the adequacy of the former as aerodynamic tracers.

\section{Conclusions}

The use of neutrally buoyant or slightly buoyant HFSB is proposed as a possible approach to solve the problem of lack of tracer particles in the vortex core precluding detailed velocity measurements by particle image velocimetry. The particles density relative to that of the air is the key parameter that determines whether the tracer particles are centrifuged outside of the vortex or accumulated at its center. Experiments conducted on the leading edge vortex of a slender delta wing assess the capability of HFSB to populate the vortex core. Neutrally buoyant HFSB appear distributed rather homogeneously within the leading edge vortex region. In contrast fog droplets are visibly centrifuged outside the vortex core and produce a "black hole". As a result, the correlation signal-to-noise ration obtained using fog droplets as tracers for stereo-PIV suffers from drop-out in the vortex core. The problem is circumvented with neutrally buoyant HFSB that yield a reliable velocity measurement in the vortex core as well as in the outer region.
The experiments illustrate that HFSB tracers exhibit some dispersion in their density, resulting in a population that spans tracers from the condition lighter-than-air to heavier-than-air. The former component tends to accumulate at the vortex axis and may be considered advantageous for the LEV visualization as they allow real-time inspection of the presence and location of vortex and the onset of vortex breakdown.

Acknowledgements The authors wish to thank Rakesh Yuvaraj for his support during the wind tunnel test campaign.

Open Access This article is distributed under the terms of the Creative Commons Attribution 4.0 International License (http://creativecommons.org/licenses/by/4.0/), which permits unrestricted use, distribution, and reproduction in any medium, provided you give appropriate credit to the original author(s) and the source, provide a link to the Creative Commons license, and indicate if changes were made.

\section{Appendix A: effect of vortex pressure distribution on HFSB of finite size}

Let us consider conservatively a particle tracer of $400 \mu \mathrm{m}$, a free stream velocity of $30 \mathrm{~m} / \mathrm{s}$ and $U_{\mathrm{t} \_ \text {max }}=U_{\text {inf. }}$ The diameter of the vortex is assumed to be $5 \mathrm{~mm}$.

Let us now take the case of a bubble placed at the location of the maximum pressure gradient (dashed blue line in 


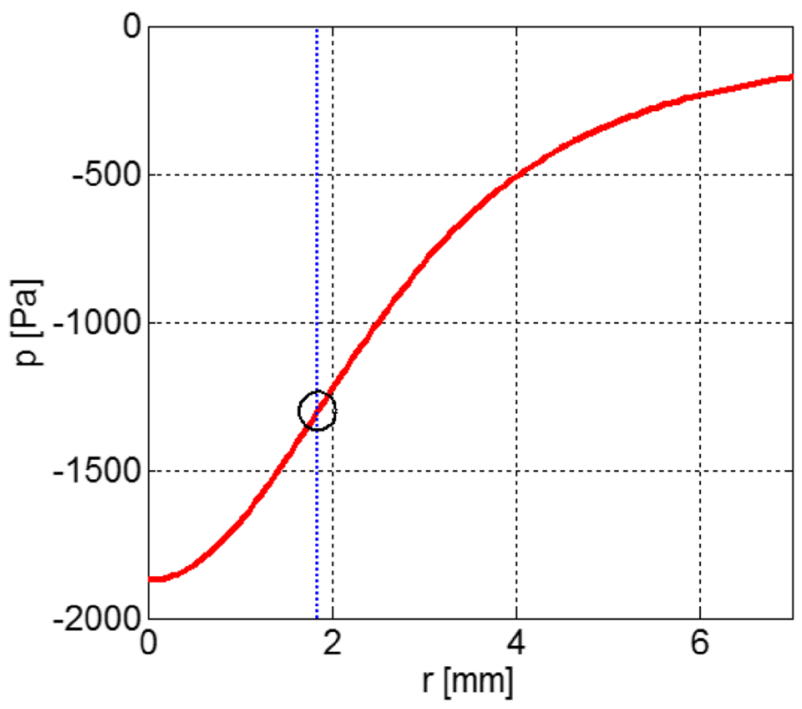

Fig. 13 Pressure distribution in a Lamb-Oseen a vortex of $5 \mathrm{~mm}$ diameter with $U_{\mathrm{t} \text { max }}=30 \mathrm{~m} / \mathrm{s}$ and location of the maximum pressure gradient (dashed line)

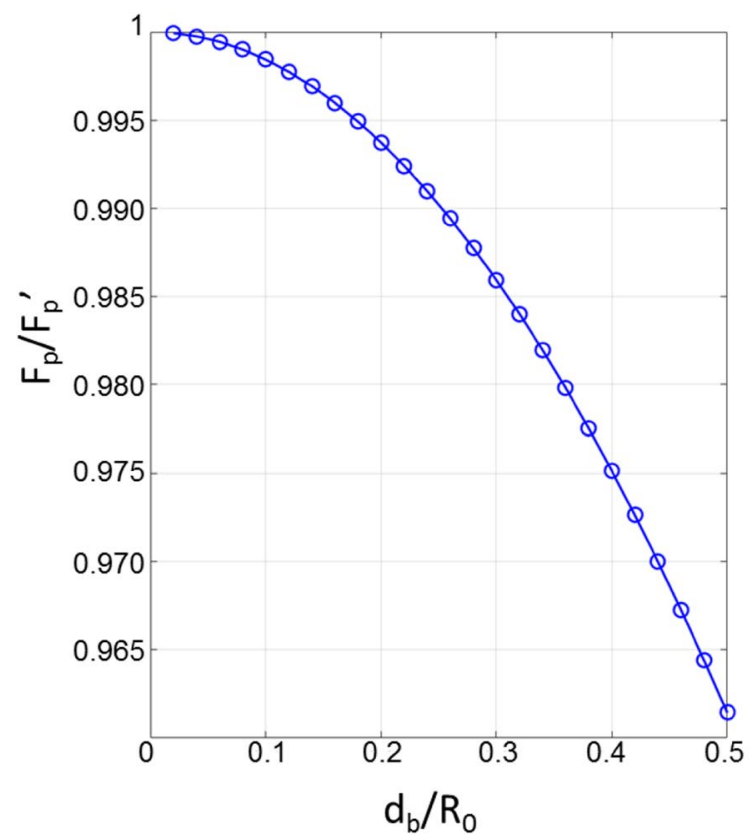

Fig. 14 Left ratio of the pressure force calculated from the pressure distribution around the bubble and the pressure force calculated from Eq. (3) using the $U_{\mathrm{t}}$ at the center of the HFSB. Right ratio of the pres-
Fig. 13, left). The tracer will experience a maximum pressure difference along its circumference of $10 \% p_{\text {core }}$, being $p_{\text {core }}$ the pressure minimum at the center of the vortex.

The pressure force $F_{\mathrm{p}}$, computed from the pressure distribution around the bubble with Eq. (6) (Fig. 13, right), is compared with the pressure force, $F_{\mathrm{p}}{ }^{\prime}$, calculated with the expression used in article and reported in Eq. (7):

$F_{\mathrm{p}}=\iint p \mathrm{~d} S$

$F_{\mathrm{p}}^{\prime}=\frac{\pi}{6} \mathrm{~d}_{\mathrm{p}}^{3} \rho_{\mathrm{f}} \frac{U_{\mathrm{t}}^{2}}{r}$.

When the tangential velocity is computed as the value at the center of the bubble, $U_{\mathrm{c}}$ (as it was computed in the article), a difference between $F_{\mathrm{P}}$ and the centrifugal force of $0.5 \%$ is retrieved, (in the present case $d_{\mathrm{b}} / R_{0}=0.16$ ), as it is shown in Fig. 14, left. The difference with $F_{\mathrm{p}}$ is even lower if the tangential velocity is computed as the mean square value in the region occupied by the bubble, $\overline{U_{t}^{2}}$ (the results are

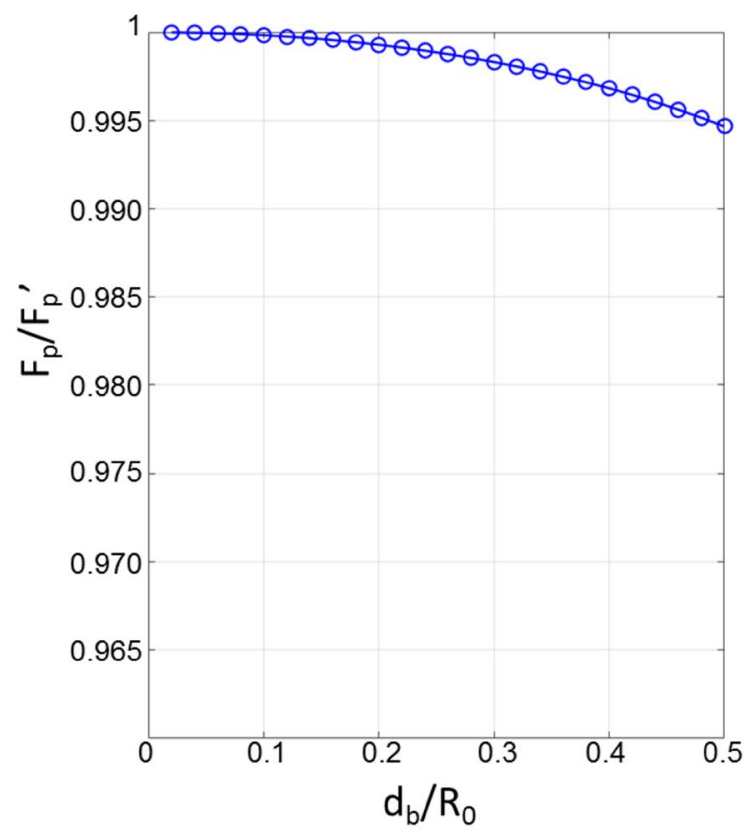

sure force calculated from the pressure distribution around the bubble and the pressure force calculated using the mean square of tangential velocity computed in the region occupied by the bubble, $U_{\mathrm{t}}^{2}$ 
shown in Fig. 14, right). The numerical simulations show that the pressure forces computed as in the article can be considered valid even for relatively large bubbles of with an approximation of $0.5 \%$.

\section{References}

Adrian RJ, Yao CS (1985) Pulsed laser technique application to liquid and gaseous flows and the scattering power of seed materials. Appl Opt 24(1):44-52

Bailey SC, Tavoularis S, Lee BH (2006) Effects of free-stream turbulence on wing-tip vortex formation and near field. J Aircr 43(5):1282-1291

Bhagwat M, Ramasamy M (2012) Effect of tip vortex aperiodicity on measurement uncertainty. Exp Fluids 53(5):1191-1202

Bosbach J, Kühn M, Wagner C (2009) Large scale particle image velocimetry with helium filled soap bubbles. Exp Fluids 46(3):539-547

Caridi GCA, Ragni D, Sciacchitano A, Scarano F (2016) HFSB-seeding for large-scale tomographic PIV in wind tunnels. Exp Fluids 53(5):1191-1202

Clancy LJ (1975) Aerodynamics. Halsted Press, New York

Conlisk AT (1997) Modern helicopter aerodynamics. Annu Rev Fluid Mech 29(1):515-567

Devenport WJ, Rife MC, Liapis SI, Follin GJ (1996) The structure and development of a wing-tip vortex. J Fluid Mech 312:67-106

Escudier M (1988) Vortex breakdown: observations and explanations. Prog Aerosp Sci 25(2):189-229

Furman A, Breitsamter C (2013) Turbulent and unsteady flow characteristics of delta wing vortex systems. Aerosp Sci Technol 24(1):32-44

Goto S (2008) A physical mechanism of the energy cascade in homogeneous isotropic turbulence. J Fluid Mech 605:355-366

Julien PY (1986) Concentration of very fine silts in a steady vortex. J Hydraul Res 24(4):255-264

Konrath R, Klein C, Schröder A (2013) PSP and PIV investigations on the VFE-2 configuration in sub-and transonic flow. Aerosp Sci Technol 24(1):22-31

Lecuona A, Ruiz-Rivas U, Nogueira J (2002) Simulation of particle trajectories in a vortex-induced flow: application to seed-dependent flow measurement techniques. Meas Sci Technol 13(7):1020

Leishman JG (1996) Seed particle dynamics in tip vortex flows. J Aircr 33(4):823-825
Lignarolo LEM, Ragni D, Scarano F, Ferreira CS, van Bussel GJW (2015) Tip-vortex instability and turbulent mixing in wind-turbine wakes. J Fluid Mech 781:467-493

Mei R (1996) Velocity fidelity of flow tracer particles. Exp Fluids 22(1): $1-13$

Meinhart CD, Wereley ST, Santiago JG (2000) A PIV algorithm for estimating time-averaged velocity fields. J Fluids Eng 122(2):285-289

Melling A (1997) Tracer particles and seeding for particle image velocimetry. Meas Sci Technol 8(12):1406

Nelson RC, Pelletier A (2003) The unsteady aerodynamics of slender wings and aircraft undergoing large amplitude maneuvers. Prog Aerosp Sci 39(2):185-248

Raffel M, Richard H, Ehrenfried K, Van der Wall B, Burley C, Beaumier P, McAlinster K, Pengel K (2004) Recording and evaluation methods of PIV investigations on a helicopter rotor model. Exp Fluids 36(1):146-156

Raffel M, Willert C, Wereley S, Kompenhans J (2007) Particle image velocimetry - a practical guide, 2nd edn. Springer, Berlin Heidelberg

Rockwell D (1993) Three-dimensional flow structure on delta wings at high angle-of-attack - experimental concepts and issues. In: 31 st aerospace sciences meeting, 550

Ruith MR, Chen P, Meiburg E, Maxworthy T (2003) Three-dimensional vortex breakdown in swirling jets and wakes: direct numerical simulation. J Fluid Mech 486:331-378

Samimy M, Lele SK (1991) Motion of particles with inertia in a compressible free shear layer. Phys Fluids 3:1915-1923

Scarano F, Ghaemi S, Caridi GCA, Bosbach J, Dierksheide U, Sciacchitano A (2015) On the use of helium-filled soap bubbles for large-scale tomographic PIV in wind tunnel experiments. Exp Fluids 56(2):1-12

Sohn MH, Chang JW (2012) Visualization and PIV study of wing-tip vortices for three different tip configurations. Aerosp Sci Technol 16(1):40-46

Spalart PR (1998) Airplane trailing vortices. Annu Rev Fluid Mech 30(1):107-138

Stanislas M, Okamoto K, Kähler C (2003) Main results of the first international PIV challenge. Meas Sci Technol 14(10):R63

Vermeer LJ, Sørensen JN, Crespo A (2003) Wind turbine wake aerodynamics. Prog Aerosp Sci 39(6):467-510

Wang C, Gao Q, Wei R, Li T, Wang J (2016) 3D flow visualization and tomographic particle image velocimetry for vortex breakdown over a non-slender delta wing. Exp Fluids 57(6):1-13

Westerweel J, Elsinga GE, Adrian RJ (2013) Particle image velocimetry for complex and turbulent flows. Annu Rev Fluid Mech 45:409-436 